\title{
MODIFIED ADAPTIVE BILATERAL FILTER FOR IMAGE CONTRAST ENHANCEMENT
}

\author{
R.Rejitha ${ }^{1}$, A.Kanagaraj ${ }^{2}$ \\ ${ }^{1}$ PG Scholar, Department of CSE, Sardar Raja college of Engineering, Alankulam, Tamil Nadu \\ ${ }^{2}$ Assistant Professor, Department of CSE, Sardar Raja college of Engineering, Alankulam, Tamil Nadu.
}

\begin{abstract}
This paper proposes a two phase theme for removing noise and improves the resolution improvement victimization Adaptive bilateral filter. Within the initial part, to get rid of total variant regularization is employed to spot pixels that square measure probably to be contaminated by noise. Within the second part, the image is rebuilt employing a specialized technique that applies solely to those chosen component candidates for improvement. The filter may be applied iteratively to effectively scale back significant noise. In terms of edge preservation and noise suppression, our rebuilt images show a big improvement compared to those rebuilt by victimization simply nonlinear filters or regularization strategies solely. It's additionally called multiple-look process. Our theme will take away background level as high as attainable and provides high resolution output compared to alternative strategies.
\end{abstract}

Keywords-Bilateral Filter, Denoising, Enhancement, Quality

\section{INTRODUCTION}

Image process is associate degree application space that needs quick realization of sure computationally intensive operations and therefore the ability of the system's developer to experiment with algorithms. High accomplishment arrangement is acceptable in Image process applications, space it ought to be alternate and experimental, so the creator will modify, tune or alter the algorithmic rule speedily and handily. Image process involves moderating a two-dimensional Image because the judge of a arrangement and outputting a custommade Image or a group of shaping reach incidental to the image. Trendy Image process tends to accredit to the agenda space the blush of day component is outlined by a wire of lay eyes on digits. However abundant techniques are at home with analog[1] and even optical pictures. Image process involves abundant transformations and techniques, sometimes no inheritable from the land area of stunning process. There are accepted geometric transformations like enlargement, measurements reduction [2], path adaptation and rotation. It's accessible to adapt the colors in pictures like acceptable contrasts or maybe reworking the Image into associate degree fully altered blush palette in line with some specific mapping system. Compositions of pictures are overtimes conducted to soak up parts from various pictures. Another breadth of absorption involves interpolation. Basically, pictures retrieved in some contexts are spread with missing pixels. Accepted techniques absorb artlessly ciphering the missing pixels supported the blush of the adjoining accepted pixels. Sweetening operations are agitated dead set advance the interpretability of the Image by accretion credible adverse a neighborhood of varied look within the scene. As a picture sweetening technique typically drastically alters the first numeric information, it's ordinarily used just for visual interpretation and not for any numeric analysis.

\section{IMAGE RESOLUTION}

Resolution is a capability of associate degree imaging system to record fine details in a very distinguishable manner. An operating data of resolution is important for understanding each sensible and abstract details of remote sensing. Together with the particular positioning of spectral bands, they're of dominant importance in crucial the suitableness of remotely detected information for a given applications. the most important characteristics[3][4] of imaging remote sensing instrument operational within the visible and infrared spectral region are delineate in terms as Spectral resolution, Radiometric resolution, special resolution and Temporal resolution. Spectral Resolution refers to the breadth of the spectral bands. These spectral characteristics outline the spectral position and spectral sensitivity so as to differentiate materials. There's a trade-off between spectral resolution and signal to noise. Radiometric Resolution [6] or radiometric sensitivity refers to the quantity of digital levels accustomed specific the information collected by the sensing element. It's normally expressed because the range of bits must store the most level. Here additionally there's a trade-off between radiometric resolution and signal to noise. There's no purpose in having a step size but the background level within the information. A low-quality instrument with a high background level would essentially, therefore, have a lower radiometric resolution compared with a high-quality[8], high signal-tonoise-ratio instrument. Additionally higher radiometric resolution could conflict with information storage and transmission rates. 


\section{IMAGE FILTER}

Image filtering is helpful for several applications, together with smoothing, sharpening, removing noise, and edge detection. A filter is outlined by a kernel that may be a little array applied to every picture element and its neighbors at intervals a picture. In most applications, the middle of the kernel is aligned with this picture element, and may be a square with an odd variety of components in every dimension. The method wont to apply filters to a picture is understood as convolution, and should be applied in either the spacial or frequency domain. at intervals the spacial domain, the primary a part of the convolution method multiplies the weather of the kernel by the matching picture element values once the kernel is targeted over a picture element. The weather of the ensuing array is averaged, and also the original picture element price is replaced with this result. The filter perform performs this convolution method for a whole image.

\subsection{Modified Adaptive Bilateral filter}

In our proposed method modified adaptive bilateral filter smoothes pictures whereas conserving edges, by suggests that of a nonlinear combination of close image values. And although bilateral filtering may be a nonlinear technique it's non-iterative, 'local' and straightforward. The nonlinearity arises attributable to the nonlinear relationship of picture element values of a picture. Our proposed bilateral filters comprise of 2 element filters: a domain filter and a variety filter. Domain filter element refers to the normal low-pass filter wont to average values of the image. Our implementation of the domain filter utilizes a Gaussian blur kernel for filter weights. This adaptive quality is illustrated within the figures below. Bilateral filter is that the resultant product of the domain and vary filters, which ends in an averaging of image pixels supported special and measuring closeness. This is often the central plan underlying the bilateral filtering interpolation technique.

Consider an image $H(1), H(2) \ldots H(n)$ where $n$ is the present time point and performs the following steps:

1) For index $\mathrm{k}$ sufficiently large, carry out the Gaussian model on $\{H(1), H(2) \ldots H(n)\}$.

2) Retain the coefficient values as well as the smooth values for the $\mathrm{k}$ time point only: $\mathrm{D}_{1}(\mathrm{k}), \mathrm{D}_{2}(\mathrm{k}) \ldots \mathrm{D}_{5}(\mathrm{k})$. The summation of these values gives $\mathrm{H}(\mathrm{k})$.

3) If $\mathrm{k}<\mathrm{n}$, set $\mathrm{k}=\mathrm{k}+1$ and go to Step 1 .

This process produces an additive decomposition of the signal $\mathrm{H}(\mathrm{k}), \mathrm{H}(\mathrm{k}+1), \ldots, \mathrm{H}(\mathrm{n})$, The result of the complete transformation, $\mathrm{T}$, is composed of 4 new sub-images, which correspond to the blurred image, and the vertical, diagonal, and horizontal differences between the original image and the blurred image. The blurred representation of the image removes the details, which are represented separately in the other three images, in a manner that produces a sparser representation overall, making it is easier to store and transmit.
Bilateral filter adaptively smoothes an input image over neighboring pixels with edge detection capability. Throughout this section the input image is denoted $\mathrm{f}$ while the output image is denoted $\mathrm{g}$. The output image content at pixel $(\mathrm{x}, \mathrm{y})$ is computed as a weighted average of the input image contents of all pixels within a $(2 \mathrm{~W}+1)$-by- $(2 \mathrm{~W}+1)$ window around the pixel $(x, y)$, where $\mathrm{W}$ is predefined.

From the Gaussian model its weight at or near the center and exponentially diminishes away from the center. Thus, both the domain filter kernel and the range filter kernel have been designed with the Gaussian as follows

$$
H(f(\vec{z}), f(\vec{\zeta}))=\exp \left(-\frac{s_{r}(f(\vec{z}), f(\vec{\zeta}))^{2}}{2 \sigma_{r}^{2}}\right)
$$

After that we apply smoothing process to the pixels that area unit in shut geometric proximity have similar contents. Thus, it's assumed safe to average over shut pixels. However, this central plan breaks down at the 'edges' of a picture. During this context, 'edges' ask those points on a picture wherever there are a unit discontinuities or sharp contrasts between pixels content and its immediate neighbor's. The bilateral filter accounts for the sides by weight pixels supported their photometrical similarity additionally to geometric proximity. Therefore, the bilateral filter kernel may be a composite kernel consisting of a site filter kernel and a variety filter kernel. The domain filter kernel weights pel contents supported geometric proximity to the middle pel whereas the vary filter kernel weights the pel contents supported photometrical similarity to the middle pel. The mathematician perform may be a natural candidate for implementing such a weight theme. The mathematician mask contains parts determined by a mathematician perform. This convolution brings the worth of every pel into nearer harmony with the values of its neighbors. In general, a smoothing filter sets every pel to the common worth, or a weighted average, of itself and its near neighbors; the mathematician filter is simply one potential set of weights. Smoothing filters tend to blur a picture, as a result of pel intensity values that area unit considerably higher or less than the encompassing neighborhood would "smear" across the world. As a result of this blurring, linear filters area unit rarely employed in follow for noise reduction; they're, however, typically used because the basis for nonlinear noise reduction filters.

\section{EXPERIMENTAL RESULTS}

Experimental results show that our method performs much better than median-based filters or the edge-preserving regularization methods. Even at a very high noise level, the texture, details and edges are preserved accurately 


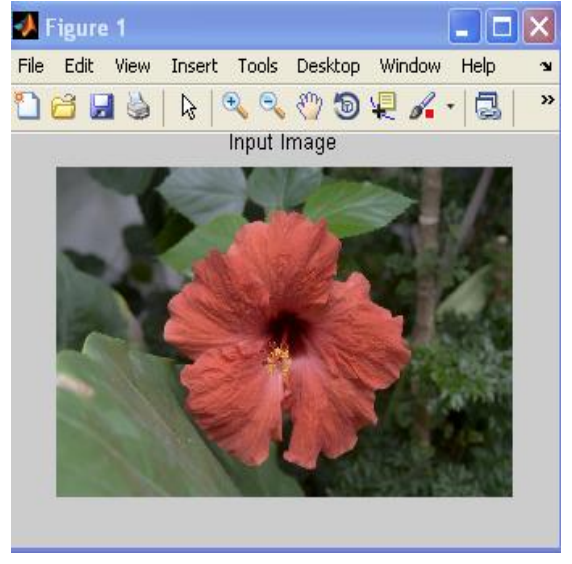

Fig 1: Input image

The above input image is given to the system for enhancement process.

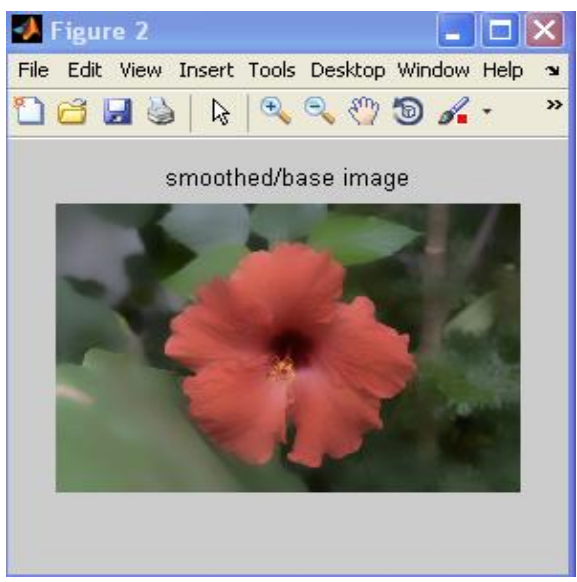

Fig 2: Smoothed image

The input image is given to the system for smoothing purpose by using smoothing filter.

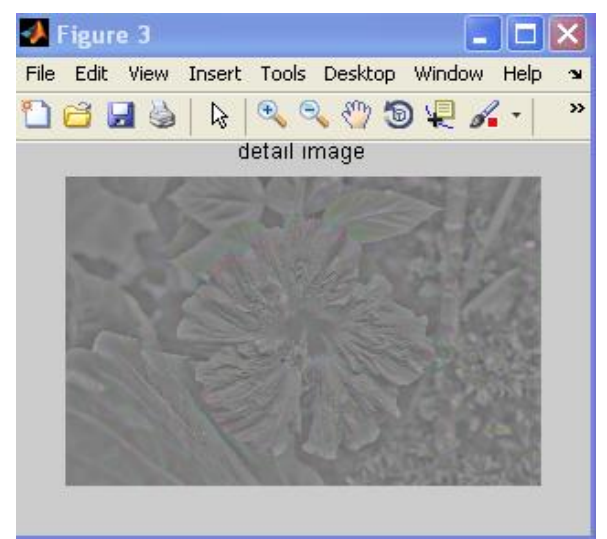

Fig 3: Detail image
Then the image features are exposed after smoothing process.

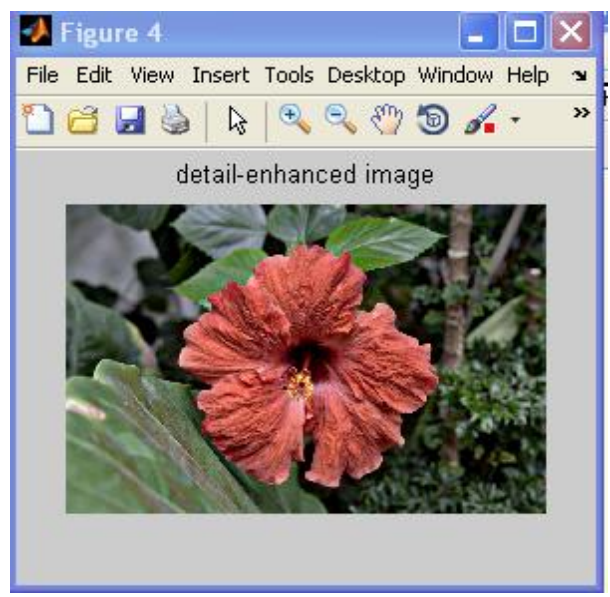

Fig 4: Enhanced output image

Image quality is a characteristic of an image that measures the Image quality may be a characteristic of a picture that measures the perceived image degradation generally, compared to a perfect image. By shaping image quality in terms of a deviation from the perfect state of affairs, quality measures become technical within the sense

Table 1: Quality Measure

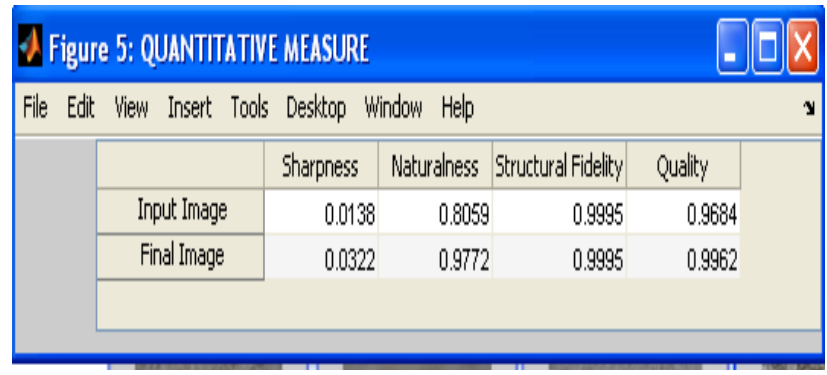

The above Table shows the image pixel classification which aims at identifying points in a digital image at which the image brightness changes sharply or, more formally, has discontinuities.

\subsection{Performance Comparison}

Below figure shows that performance Comparison graph of our proposed methods with various other methods 


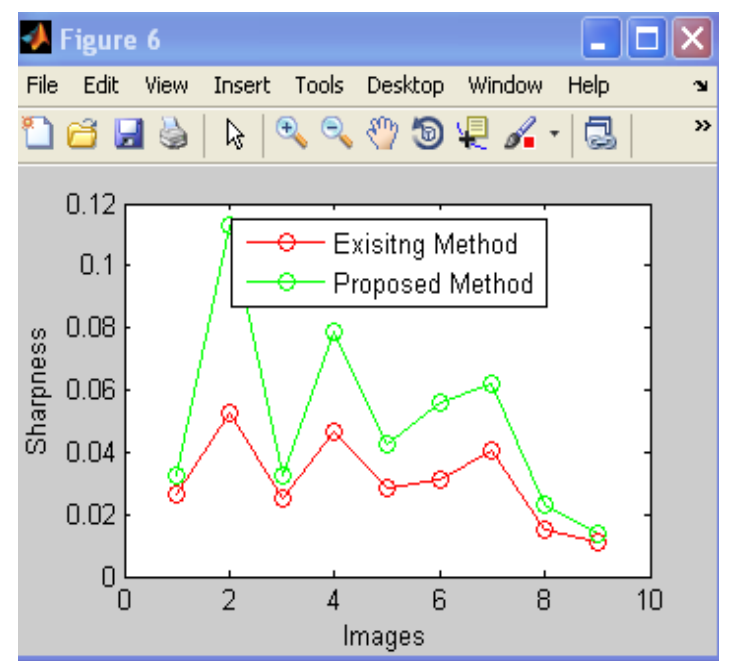

Fig 5: Sharpness Comparison

Sharpness determines the number of detail a picture will convey. System sharpness is full of the component count and anti-aliasing filter. Within the field, sharpness is full of camera shake, focus accuracy, and part disturbances. Lost sharpness will be remodeled by sharpening,

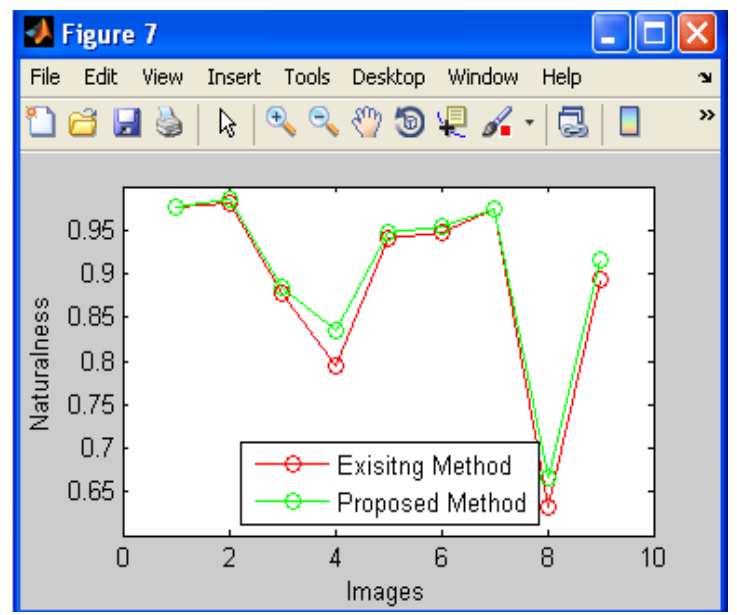

Fig 6.Naturalnesss Comparison

Color accuracy is an important image quality factor. The most accurate color isn't necessarily most pleasing. Nevertheless it is important to measure a camera's color response, its color shifts, saturation, and the effectiveness of its white balance algorithms.

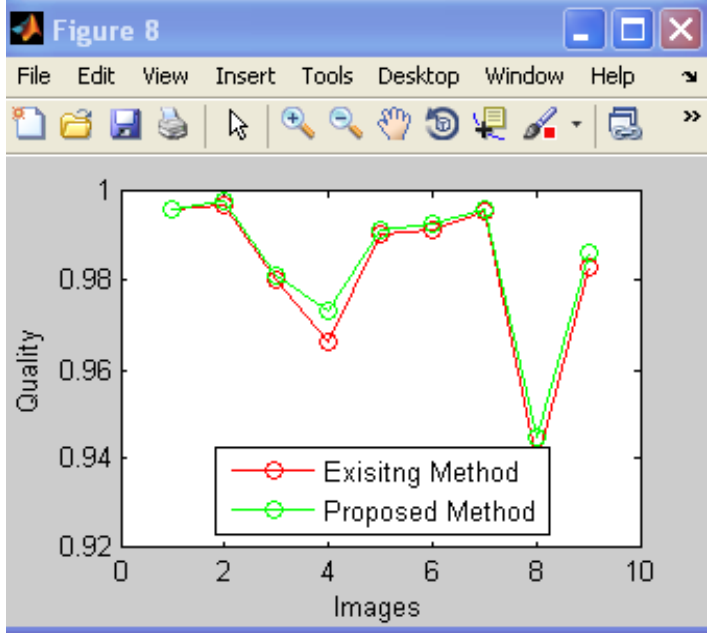

Fig 7.Quality Comparison

\section{CONCLUSIONS}

This paper projected a brand new means of denoising and enhances the image victimization modified adaptive bilateral filter. Its main feature is that it distinguishes between native variations as a result of noise and as a result of image structures, victimization deconvolution estimation. In addition, the form of the membership functions is customized per the remaining quantity of noise when every iteration. Experimental results show the practicableness of the new filter.

\section{REFERENCES}

[1]. G. Aubert and J. Aujol, "A variational approach to remove multiplicative noise," SIAM J. Appl. Math., vol. 68, no. 4, pp. 925-946, 2008.

[2]. M. Bazaraa, H. Sherali, and C. Shetty, Nonlinear Programming: Theory and Algorithms. Hoboken, NJ: Wiley, 1993.

[3]. A. Beck and M. Teboulle, "A fast iterative shrinkagethresholding algorithm for linear inverse problems," SIAM J. Imag. Sci., vol. 2, pp. 183-202, 2009

[4]. J. Bioucas-Dias and M. Figueiredo, "An iterative algorithm for linear inverse problems with compound regularizers," in Proc. IEEE Int. Conf. Image Processing, San Diego, CA, 2008, pp. 685-688.

[5]. J. Bioucas-Dias and M. Figueiredo, "Total variation restoration of speckled images using a split-Bregman algorithm," in Proc. IEEE Int. Conf. Image Processing, Cairo, Egypt, 2009, pp. 3717-3720.

[6]. J. Bioucas-Dias and M. Figueiredo, "A new TwIST: Twostep iterative shrinkage/thresholding algorithms for image restoration," IEEE Trans. Image Process., vol. 16, no. 12, pp. 2980-2991, Dec. 2007.

[7]. J. Bioucas-Dias, T. Silva, and J. Leitão, "Adaptive restoration of speckled SAR images," in Proc. IEEE Int. 
Geoscience and Remote Sensing Symp., 1998, vol. I, pp. 1923.

[8]. A. Chambolle, "An algorithm for total variation minimization and applications,"J. Math. Imag. Vis., vol. 20, pp. 89-97, 2004.

[9]. T. Chan, S. Esedoglu, F. Park, and A. Yip, "Recent developments in total variation image restoration," in Mathematical Models of Computer Vision. Berlin, Germany: Springer-Verlag, 2005.

[10]. T. Chan, G. Golub, and P. Mulet, "A nonlinear primaldual method for total variation-based image restoration," SIAM J. Sci. Comput., vol. 20, pp. 1964-1977, 1999.

\section{BIOGRAPHIES}

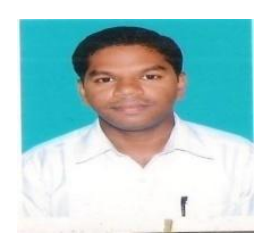

Prof.A.Kanagaraj, received B.E degree from EEE department, Government college of Engineering Bargur under Anna University, and M.E degree from CSE department, Government college of Engineering Tirunelveli from Anna

University, Tirunelveli. His current research interests include image enhancement and image denoising.

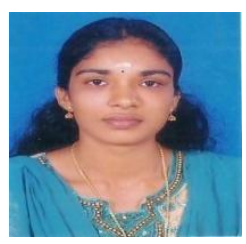

Rejitha.R received B.Tech degree in computer Science and Engineering from Baselious Mathews II College of Engineering under Kerala University and M.E degree in Computer Science and Engineering from Sardar Raja College of Engineering, Tirunelveli under Anna University. Her current research includes image enhancement, denoising and high dynamic range image processing. 